Musées, Patrimoine et Culture scientifiques et techniques

$166 \mid 2016$

juillet-août 2016

\title{
Faire vivre la culture en milieu pénitentiaire
}

\section{Morrad Benxayer}

URL : http://journals.openedition.org/ocim/1679

DOI : $10.4000 /$ ocim. 1679

ISSN : 2108-646X

\section{Éditeur}

OCIM

Édition imprimée

Date de publication : 1 juillet 2016

Pagination : 20-27

ISSN : 0994-1908

Référence électronique

Morrad Benxayer, « Faire vivre la culture en milieu pénitentiaire », La Lettre de I'OCIM [En ligne],

166 | 2016, mis en ligne le 01 juillet 2017, consulté le 19 avril 2019. URL : http://

journals.openedition.org/ocim/1679; DOI : 10.4000/ocim.1679

Ce document a été généré automatiquement le 19 avril 2019

Tous droits réservés 


\title{
Faire vivre la culture en milieu pénitentiaire
}

\author{
Morrad Benxayer
}

Sculptures d'ombre en fer découpé et peintures numériques d'Hervé Di Rosa parmi les œuvres des détenus dans l'exposition Dehors-Dedans, présenté du 14 au 17 juin 2015 au musée de l'Hospice Saint-Roch à Issoudun.

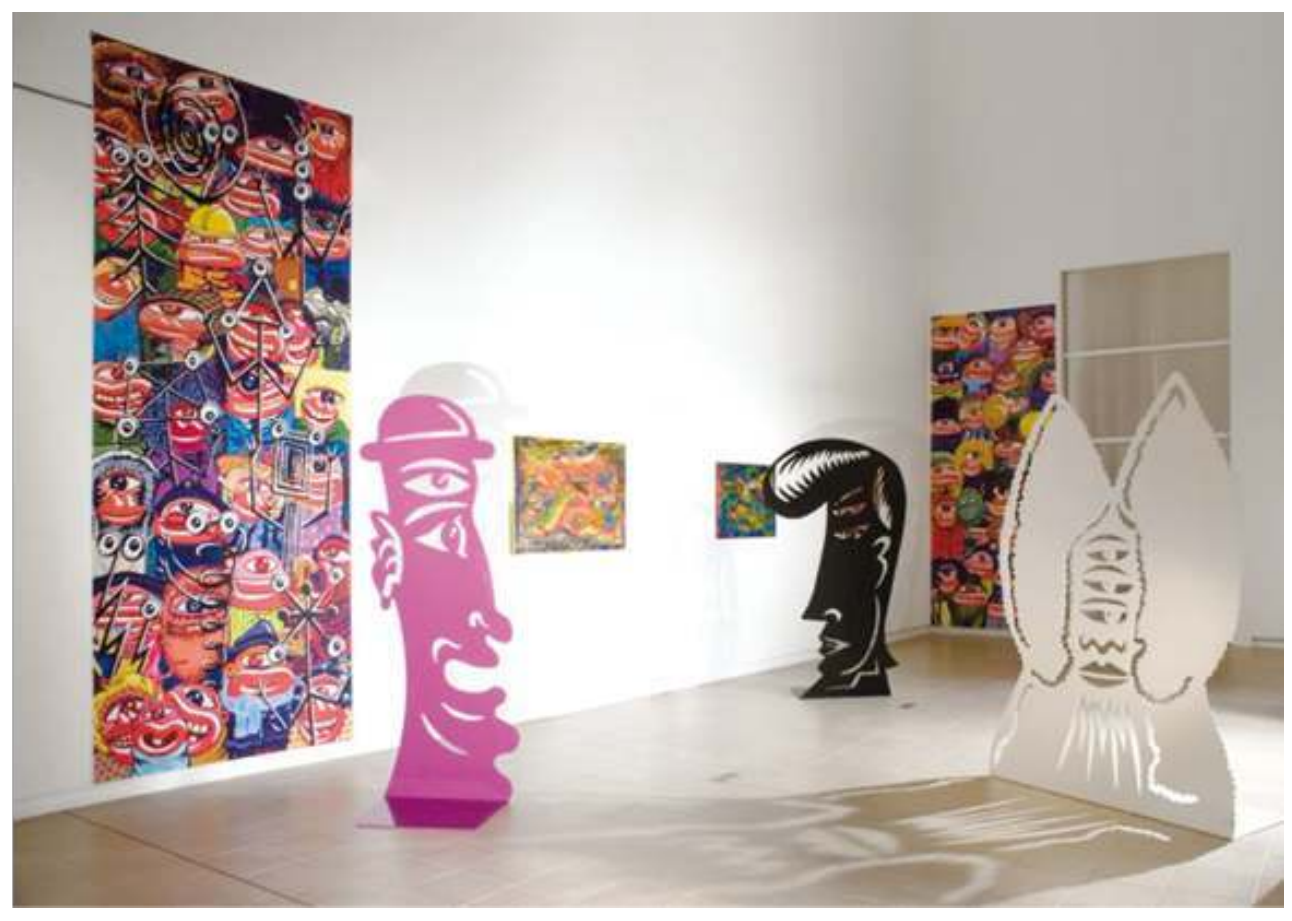

(c) Alberto Ricci/ADAGP, Paris 2015

Il existe, entre les acteurs de la Justice et de la Culture, une différence dans la perception des projets culturels. L'action culturelle est ainsi identifiée par l'institution judiciaire comme un vecteur d'ouverture et d'échange, d'éducation et de citoyenneté, pouvant contribuer à la revalorisation de l'estime de soi, à la maitrise des fondamentaux, à 
l'approfondissement des savoirs de base et à l'acquisition des compétences professionnelles. Pour les professionnels de la culture, il s'agit particulièrement d'affirmer la fonction sociale des établissements culturels - patrimoniaux ou artistiques en agissant auprès de publics qui n'ont pas accès à la culture ou qui en ont été privés ou éloignés.

2 Le Département de la politique des publics, de la Direction générale des patrimoines, et la Direction de la formation continue, de l'École nationale de l'administration pénitentiaire ${ }^{1}$, ont organisé du 17 au 21 novembre 2014 à Agen, une formation ayant pour objectif de permettre à des professionnels de la culture, agissant dans le champ patrimonial (musées, archives, villes et pays d'art et d'histoire), et à des personnels pénitentiaires de développer une culture et une méthodologie de projets communes.

Si l'accès à la culture des personnes placées sous main de justice est un droit fondamental, reconnu tant au niveau national ${ }^{2}$ qu'au niveau international ${ }^{3}$, et réaffirmé par les trois protocoles d'accord signés en 1986, 1990 et 2009 entre le ministère de la Justice et le ministère de la Culture et de la Communication, la mise en œuvre à leur adresse d'une politique culturelle de qualité ne va pas sans difficultés, malgré la diversité des projets réalisés.

Un état des lieux des actions menées par les établissements patrimoniaux en faveur de la population pénale a été commandé par la Direction générale des patrimoines en 2010. Celui-ci a relevé un certain nombre de freins à la réussite des projets 4 . Tout d'abord, l'extrême dissemblance des modes organisationnels des projets culturels et l'impréparation des personnels pénitentiaires. Puis, l'insuffisante connaissance du cadre institutionnel instauré par le dernier protocole d'accord du 30 mars 2009 ainsi que le peu de visibilité des expériences réalisées et, par conséquent, la déperdition d'une richesse de solutions et de dispositifs qui pourrait inspirer d'autres situations et structures.

5 À l'évidence, la difficulté principale qui émerge dans la construction des projets résulte d'une méconnaissance du monde patrimonial et du monde pénitentiaire, par chacun des acteurs et, notamment, de leur environnement, fonctionnement et mécanismes. L'état des lieux met également en exergue la question des motivations des acteurs impliqués. Il relève le caractère individuel des engagements des professionnels dans les projets culturels. Ce constat sous-entendrait qu'il n'existe pas de réflexion politique, à l'échelle nationale, qui dépasserait la seule profession de foi déclarative.

Pour pallier ces faiblesses, le recours aux seules solutions méthodologiques suffirait-il ? Si solutions il y a, quelles sont-elles? Ne s'agirait-il aussi pas de faire évoluer les pratiques professionnelles à l'œuvre dans les projets en interrogeant les représentations des acteurs sur le rôle de la culture et le sens de la peine?

7 L'expérimentation menée par le Département de la politique des publics et l'École nationale d'administration pénitentiaire (Enap) vise à proposer quelques réponses à ces questions et à en inventer d'autres en faisant interagir les stagiaires. Elle ne prétend pas non plus mettre entre les mains des acteurs impliqués dans des projets culturels destinés aux personnes placées sous main de justice des solutions toutes faites. 
Hervé di Rosa et Jean-Marc Le Bruman, artiste-peintre, coordonnateur culturel du SPIP-FOL de I'Indre dans l'exposition Dehors-Dedans, 21 novembre 2014

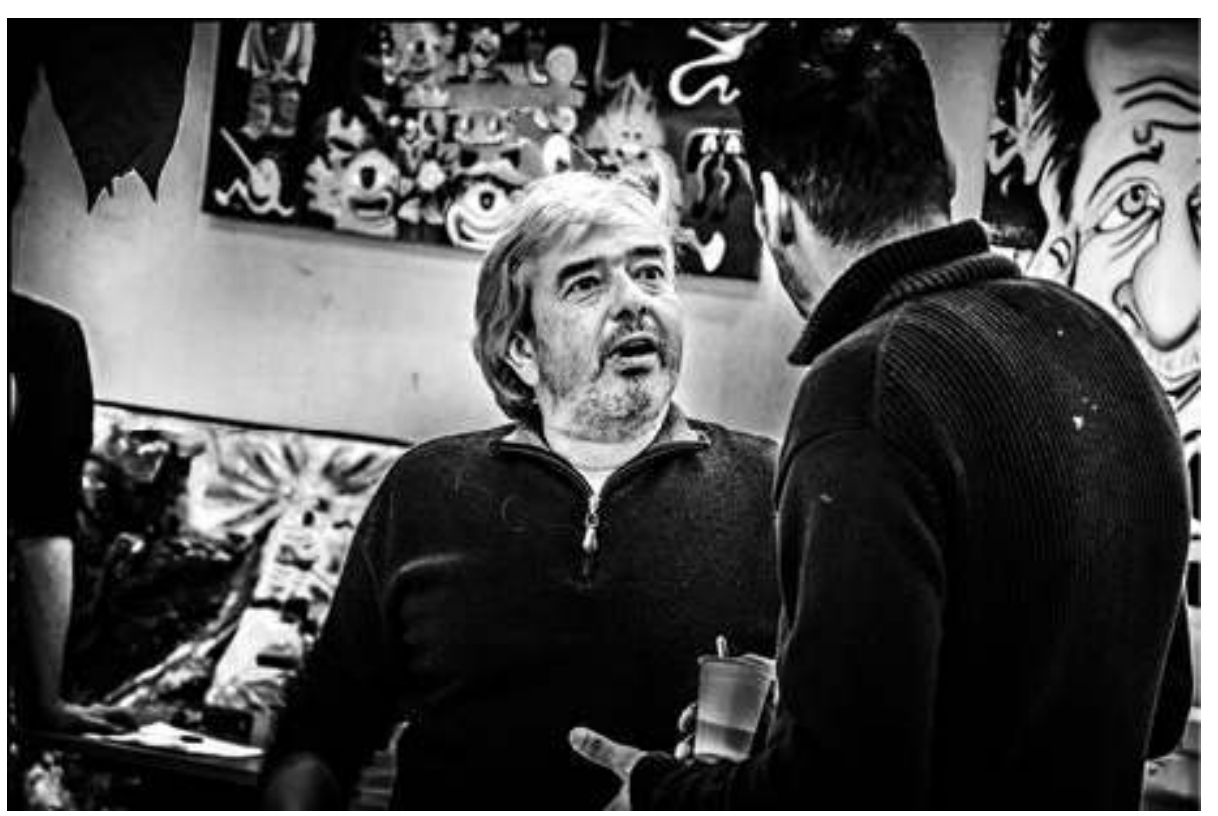

(c) Gilles Froger disposent d'une première expérience de projet, ou, à tout le moins, qu'ils ambitionnent de réaliser un projet culturel. C'est la raison pour laquelle il a été exigé de leur part, au moment de leur inscription au stage, d'avoir élaboré ou contribué à la mise en œuvre d'actions culturelles tant en milieu fermé qu'ouvert ${ }^{5}$, ou avoir un projet en cours de développement.

14 Les objectifs pédagogiques, élaborés avec l'équipe de la Direction de la formation continue de l'Enap, visent, d'une part, à faire découvrir et comprendre les patrimoines, pour permettre d'ancrer les actions culturelles dans les missions des services pénitentiaires d'insertion et de probation (SPIP) et des établissements pénitentiaires, 
d'autre part, à resituer l'action des structures patrimoniales et des structures judiciaires dans le cadre du protocole Culture/Justice. La formation doit ainsi permettre de comprendre les enjeux de démocratisation culturelle inscrits dans les politiques et les missions des ministères signataires du protocole d'accord. Elle doit, autant que faire se peut, déboucher sur une réflexion commune sur la prise en charge des personnes condamnées et la place de la culture dans la société. Les contenus comprennent, outre la découverte de la médiathèque de l'Enap (dont le centre de ressources sur l'histoire des crimes et des peines), la visite du musée archéologique d'Eysses (Lot-et-Garonne) et de l'atelier de restauration archéologique situé dans le centre de détention d'Eysses, une phase didactique autour de la construction d'un projet culturel patrimonial (cadre institutionnel, méthodologie de projet, élaboration d'une convention, méthodologie d'évaluation) et une phase collaborative (illustrations et témoignages autour des projets du musée Fabre de Montpellier et du musée des Beaux-Arts de Nantes) durant laquelle une interactivité s'instaure avec les intervenants. Des ateliers de mise en pratique des projets des stagiaires, permettant de mettre à profit les méthodes présentées par les intervenants mais aussi les compétences des stagiaires, complètent la formation. La restitution de ces projets clôt la formation. Elle est l'occasion pour les stagiaires et les formateurs d'examiner les atouts et les faiblesses des actions projetées.

L'intérêt d'une telle formation est qu'elle mobilise les formateurs et les stagiaires pendant près de cinq jours, dans une sorte de microcosme très enrichissant pour chacun. Les stagiaires comme les intervenants extérieurs sont logés sur le campus de l'Enap et se retrouvent également au moment des repas et des pauses. Au-delà des séances de travail, une connivence se crée, permettant des échanges sur les parcours des uns et des autres. La découverte des univers professionnels de chacun, notamment de leur spécificité, contribue également à mettre à bas les a priori, voire les réticences à engager des actions communes.

17 Trois dispositifs de médiation culturelle vont servir de supports sur les plans méthodologique et de l'illustration. 
Peintures de détenus exposées en 2013 au Centre pénitentiaire de Nantes.

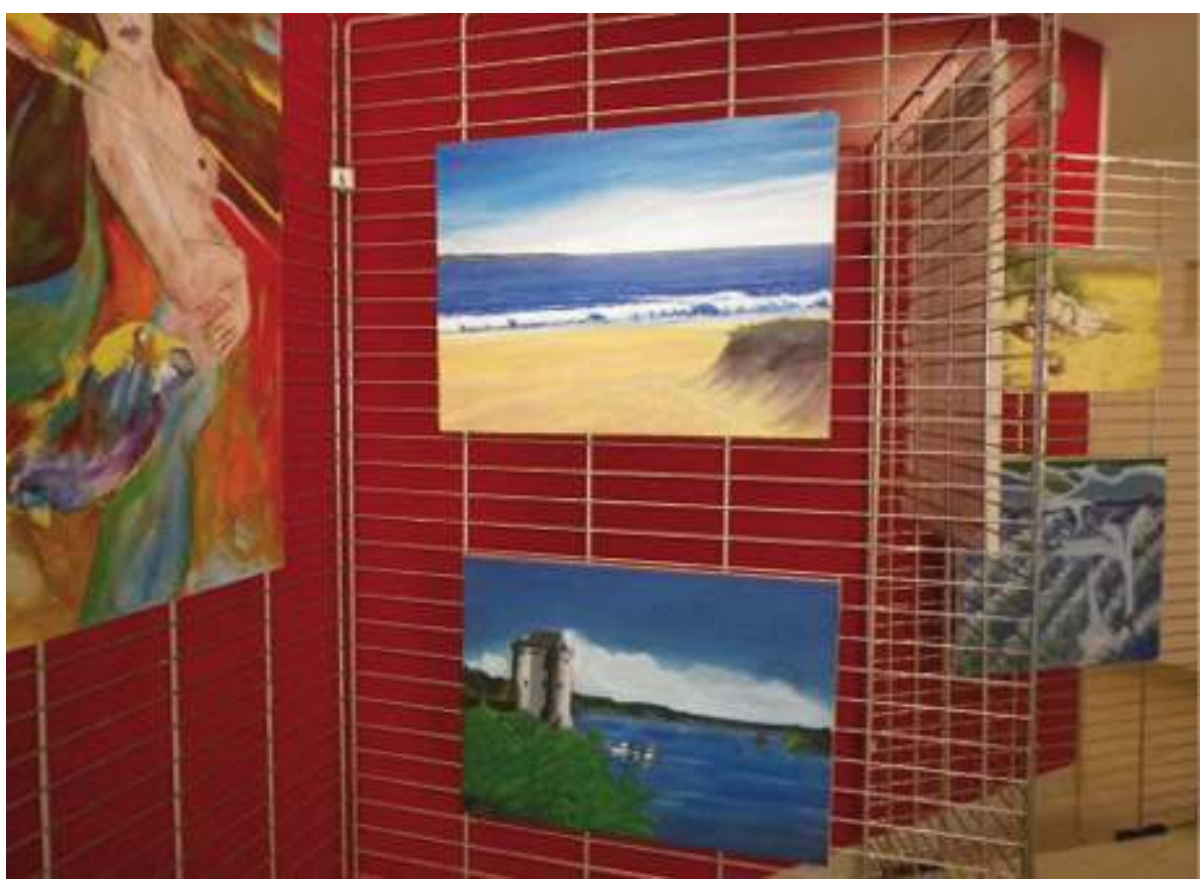

(c) Musée des Beaux-Arts de Nantes Montpellier, présenté par Céline Peyre, responsable du service des publics, et Claudine Doutremepuich, chargée de mission culture du SPIP 34 (Hérault). Cette expérience, qui s'est déroulée durant le premier semestre 2013, s'inscrit plus largement dans un dispositif, intitulé Musée Citoyen, mis en place depuis 2008 par les deux partenaires, avec le soutien de la Direction régionale des Affaires culturelles du Languedoc-Roussillon. Le projet a pour objectif de contribuer à la future réinsertion des détenus de la maison d'arrêt de Villeneuve-lès-Maguelone, il permet ainsi aux personnes incarcérées en fin de peine d'appréhender le musée et ses collections tout en développant un lien social. Il repose sur un principe : ce sont les détenus volontaires qui viennent au musée et non l'inverse. Ce principe méthodologique fait partie d'un programme mené par le réseau des musées-franco-américains Frame, Talking Arts, dans lequel est impliqué le musée Fabre. Le projet a concerné une dizaine de détenus qui, bénéficiant d'une permission de sortie du juge d'application des peines, se sont rendus au musée, une fois par semaine pendant cinq semaines, accompagnés de référents. L'intention affichée pour cette expérience était de rendre chaque participant autonome face aux œuvres choisies en lui donnant l'objectif et la capacité de témoigner aux autres son propre vécu. À une première préparation en maison d'arrêt, succèdent cinq séances de travail au musée de $1 \mathrm{~h} 30$ chacune. Les quatre premières favorisent l'appropriation du lieu et de ses collections, avec l'aide d'une guideconférencière mais aussi avec un plasticien qui dirige des ateliers de pratiques artistiques durant lesquels les détenus sont amenés à observer des œuvres reproduites sur des cartes postales, à échanger leurs impressions, à prendre des notes écrites ou graphiques, et à choisir un thème ou un sujet leur permettant de produire à leur tour des œuvres plastiques. Après s'être approprié musée et collections, les détenus peuvent partager leur regard singulier sur les œuvres avec leur famille ou leur proche, conviés lors de la dernière séance. Le témoignage de cette expérience a été intégré dans le dispositif des 
audio-guides du musée, permettant au visiteur ordinaire de trouver dans le récit des détenus une vision des collections et de l'établissement moins spécialiste mais empreinte d'intime et d'émotion, et, d'une certaine manière, le témoignage d'une rencontre entre des citoyens et l'art. L'originalité du projet tient en particulier dans la possibilité offerte aux détenus de sortir plusieurs fois de la maison d'arrêt pour aller au musée Fabre. Pour la majeure partie des projets patrimoniaux destinés au public carcéral, la sortie au musée reste quand même une exception. Par ailleurs, Céline Peyre et Claudine Doutremepuich insistent sur le caractère fragile que revêt ce type de projet s'il n'est pas soutenu par les instances décisionnelles, du côté de l'institution patrimoniale comme du côté de l'institution pénitentiaire.

19 "Plaisirs de l'eau, plage et loisirs dans la première moitié du $20^{\mathrm{e}}$ siècle" est le deuxième projet exposé aux stagiaires, voire expérimenté puisque la médiatrice en charge du dispositif, Christel Nouviale, au service des publics du musée des Beaux-Arts de Nantes, et la plasticienne Alice Ferré, ayant participé au projet, impliquent les stagiaires dans une simulation d'atelier de pratiques artistiques pour leur faire toucher, en quelque sorte, du doigt la méthode. Le contexte de ce projet est l'ouverture à Nantes de l'exposition Plaisirs de l'eau..., en février 2013, présentant une sélection d'œuvres issues des collections du musée autour des thèmes de la plage et des loisirs. Sujet familier, fédérateur, intergénérationnel et, à l'évidence, concernant toutes les origines sociales. La Ligue de l'enseignement FAL 44, déjà engagée, avec le SPIP (service pénitentiaire d'insertion et de probation) de Loire-Atlantique, dans une dynamique partenariale avec le service des publics du musée afin d'œuvrer à la création de nouvelles passerelles entre le Centre pénitentiaire de Nantes et l'institution culturelle, saisit cette opportunité pour demander au service des publics de mettre en place un programme d'activités artistiques et culturelles ayant pour but de consolider les liens entre les personnes détenues et leurs familles. Ces activités ont lieu majoritairement dans les locaux du Centre pénitentiaire (Centre de détention et maisons d'arrêt Hommes et Femme) mais avec une particularité : l'association des familles au processus de création artistique. Concrètement, le projet est construit en plusieurs étapes. Tout d'abord, une phase préparatoire menée avec la médiatrice du musée, au moyen d'une vidéo-projection de l'exposition, dans tous les lieux du centre pénitentiaire. Ensuite, des ateliers hebdomadaires (peinture et arts plastiques) conduits par un peintre et une plasticienne, destinés à initier les détenus aux techniques artistiques. Inspirés par les œuvres de l'exposition et, plus largement, par l'histoire de l'art, les détenus réalisent des productions artistiques (dessins, toiles personnelles, triptyque collectif...) et les exposent dans les lieux d'accueil des familles qui se rendent au parloir.

Deux ateliers spécifiques viennent compléter le dispositif : à la Maison d'arrêt hommes, un atelier de mosaïque, destiné à initier les détenus aux techniques de mosaïque, et sur tous les sites du Centre pénitentiaire, un atelier "cartes postales" - pour rester dans le ton estival de l'exposition - conduit par une plasticienne. Les cartes postales produites par les détenus, en résonance avec l'exposition, servent d'invitations qui seront adressées aux familles et aux proches des détenus pour une visite commentée de l'exposition, par la médiatrice du service des publics, et une participation à un atelier similaire, cette fois-ci au musée. Les publics invités réalisent des cartes postales qu'ils renvoient à leurs proches incarcérés. Effet miroir entre ce que produisent les personnes placées sous main de justice, les œuvres exposées par le musée, les thématiques communes à toutes les catégories de publics, qu'ils soient à l'extérieur du centre pénitentiaire ou à l'intérieur, le 
dialogue esthétique créé entre les détenus et leurs familles, cette dynamique du partage a mobilisé une soixantaine de personnes pendant près de trois mois. L'absence d'un cadre conventionnel et de perspective comme de prospective amoindrit néanmoins ce projet qui aurait gagné à s'inscrire dans une durée plus longue, ne serait-ce que pour mesurer l'impact de l'opération sur les détenus, les personnels pénitentiaires et les acteurs culturels et sociaux.

Atelier "Cartes postales" pour les familles des détenus, dans l'exposition Plaisirs de l'eau, 2013

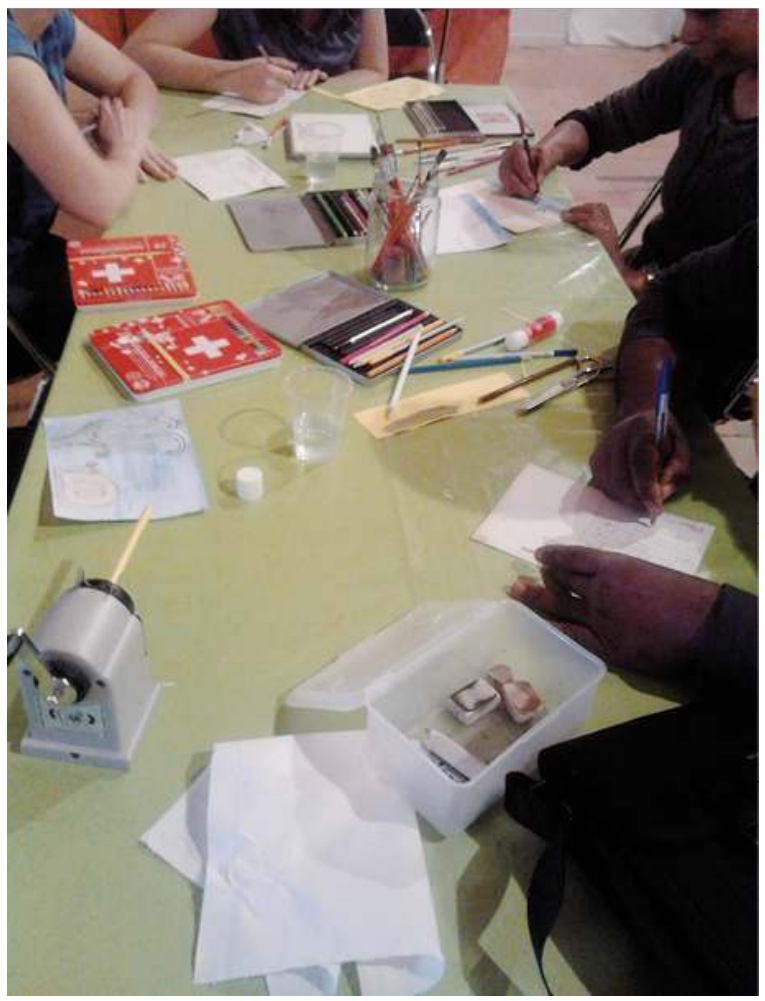

(c) Musée des Beaux-Arts de Nantes

Dernier projet illustrant la séance consacrée à l'élaboration d'une convention de partenariat, l'exposition Le Voyage, réalisée en 2013 par des détenus du Centre pénitentiaire du Sud-Francilien (CPSF), à Réaux (Seine-et-Marne), sur une initiative de la Réunion des musées nationaux-Grand Palais (Rmn-GP). Une aventure inédite où des personnes placées sous main de justice, initiées puis enthousiastes, se muent en commissaires d'exposition et en guides-conférenciers pendant près d'un mois. Le projet culturel, loin d'être seulement une opération de réinsertion, est placé sous l'angle de la démocratisation culturelle : tous les publics - détenus, personnels pénitentiaires, familles, visiteurs extérieurs - doivent avoir accès à l'art. Au total: un an de préparation, un espace d'exposition aménagé à l'intérieur du Centre pénitentiaire avec le concours de Bouygues, l'intervention des professionnels des expositions (régisseur, restauratrice, guides-conférenciers, attachée de presse, scénographe...), la mobilisation de plusieurs musées nationaux - dont le Louvre, le musée des arts asiatiques-Guimet, le musée du quai Branly, le musée national de la Marine - et territoriaux pour prêter près de 90 œuvres d'art originales, un petit journal d'exposition rédigé par les détenus, une BD intitulée Hors les murs, postfacée par le philosophe Alain Guyard, qui rend compte de cette expérience... Ce projet qui vise, outre à favoriser l'insertion par l'apport de la culture au sein du centre 
pénitentiaire, à maintenir des liens avec la société et à intégrer davantage l'établissement pénitentiaire sur son territoire, s'inscrit dans la durée au moyen d'une convention cadre associant la Direction de l'administration pénitentiaire et le ministère de la Culture, pour permettre à la Rmn-GP de poursuivre cette aventure et renouveler des actions similaires sur les territoires. Du 24 septembre au 17 novembre 2013, l'exposition Le Voyage a été vue par environ 1500 personnes: personnes détenues au centre, les familles des commissaires, le personnel pénitentiaire ainsi que leurs familles. Il convient d'ajouter à cette liste des membres de l'administration pénitentiaire et de la Rmn-GP, ainsi que les partenaires institutionnels et les journalistes. Un premier bilan met en avant les avancées obtenues avec le CPSF : mise à disposition de catalogues et d'ouvrages d'expositions dans la médiathèque du centre, diffusion d'affiches d'expositions pour habiller certaines parties de l'établissement, organisation de permissions de sorties à caractère culturel (visite entre autres de l'exposition Dynamo au Grand Palais). Il faut cependant rester attentif aux suites que souhaitent donner la Rmn-GP et le CPSF à ce dispositif partenarial.

Quelques autres exemples et un premier bilan

D'autres projets, tout aussi exemplaires que ceux présentés à la session de formation, parfois même similaires, existent. Ils sont le plus souvent le fait d'établissements muséaux. On peut citer, notamment, celui intitulé "Du processus de création à l'exposition de l'œuvre", réalisé par le musée départemental d'art contemporain du Val-de-Marne, Mac/ Val, de juin à juillet 2012, au centre pénitentiaire de Meaux-Chauconin, dans le cadre du programme de réinsertion conduit par le Service pénitentiaire d'insertion et de probation de Seine-et-Marne. 17 détenus ont pu bénéficier de conférences sur l'art contemporain (souvent réputé peu accessible au public) et d'ateliers de pratiques artistiques menés par Karim Kal, artiste photographe. Ils ont également pu se rendre au Mac/Val, une journée entière, pour visiter les expositions Vivement demain et Situation(s) et produire au cours d'un atelier des œuvres plastiques (dessins, collages, photographies...).

Au-delà des murs. De la maison centrale de Poissy au musée du Louvre, une installation sur une des façades de la maison centrale de Poissy de reproductions d'œuvres du musée du Louvre choisies par des détenus.

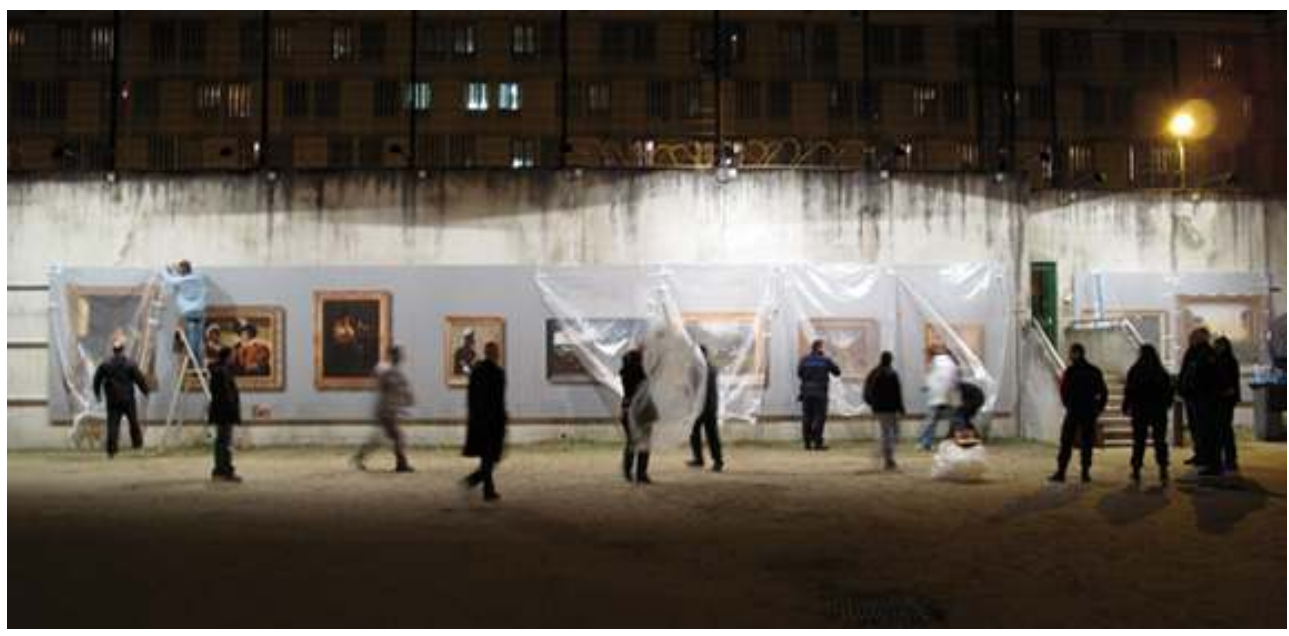

(C) A. Vaubourg/Ministère de la Justice

Avec "Au-delà des murs. De la maison centrale de Poissy au musée du Louvre", le musée du Louvre a mené une opération comparable à celle entreprise par la Rmn-GP au Centre de détention du sud-Francilien. Il s'agit d'une exposition, comprenant dix reproductions 
photographiques de peintures du musée, conçue et présentée à la maison centrale de Poissy en janvier 2011 par une équipe de 10 détenus, dans le cadre d'un programme d'éducation artistique élaboré par le Louvre en partenariat avec la maison centrale et le SPIP des Yvelines. Cette expérience a fait l'objet en novembre 2011 d'une présentation au public, pendant près d'un mois, au cœur du Louvre. Par ailleurs, un catalogue d'exposition et un film sur cette expérience ont pu être réalisés. Ce projet a, en réalité, devancé et inspiré celui de la Rmn-GP à Réau, et se pose comme une expérience fondatrice du point de vue de l'intervention muséale en prison. En revanche, le projet de la Rmn-GP se distingue de celui du Louvre parce qu'il comporte un élément important, pour ne pas dire inédit: l'existence dans le Centre pénitentiaire du Sud-Francilien d'un espace muséographique.

Autre expérience qui s'adresse, elle, aux jeunes sous protection judiciaire, l'opération " Bulles en fureur" menée par la Cité internationale de la Bande dessinée et de l'Image (CIBDI), à Angoulême. C'est un dispositif, lancé en 2001 par la Direction de la protection judiciaire de la jeunesse et la ville de Rennes, qui vise à lutter contre l'illettrisme par le biais de la bande dessinée. L'opération se déroule pendant 10 mois et mobilise près de 800 jeunes placés dans les établissements de la PJJ et dans les quartiers mineurs des centres pénitentiaires. Une douzaine de bandes dessinées d'auteurs connus et moins connus sont choisies par les partenaires de l'opération parmi la quarantaine pré-sélectionnée par la CIBDI. Elles servent de supports pédagogiques pour les équipes éducatives de la PJJ et permettent d'engager des activités multidisciplinaires où se côtoient le cinéma, la vidéo, l'art théâtral, le dessin, l'écriture, la peinture... Les BD sélectionnées sont finalement soumises à un vote des jeunes participant à l'opération et deux candidats sortent vainqueurs de cette épreuve. Ils sont récompensés lors de la fête nationale Bulles en Fureur qui se déroule chaque année, au mois d'octobre, à Rennes. Lors de cette manifestation, les jeunes qui le souhaitent ont également la possibilité de participer à des ateliers de création avec des auteurs de $\mathrm{BD}$, d'assister à des représentations, ou encore de présenter au prix Jeunes Créateurs une création artistique (pièce de théâtre, court-métrage...) réalisée à partir de la bande dessinée de leur choix. Ce dispositif se déploie dans le cadre d'une convention trisannuelle reconductible et comporte un volet évaluation.

On pourrait également citer "Un jour, une œuvre", dispositif expérimenté par le Centre Pompidou à la Maison d'arrêt du Val-d'Oise où, Michel Gauthier, conservateur du musée national d'art moderne, a accompagné durant une journée l'artiste Philippe Mayaux pour la présentation de son œuvre Les 4 z'éléments: air, eau, feu, pierre à une quarantaine de détenus. Ou encore "Résidence d'artiste" des musées de Marseille. C'est un programme d'éducation artistique, mis en œuvre en 2010 par le service des publics et destiné aux jeunes du pénitencier pour mineurs La Valentine. En 2013-2014, les jeunes détenus abordent avec un artiste le thème du portrait qu'ils expérimentent dans le cadre d'ateliers d'écriture et de peinture. Des permissions de sortie, délivrées par le juge d'application des peines, leur permettent de découvrir l'exposition "D'un visage à l'autre: Ensor, Bacon, Picasso, Hooper, Giacometti, Warhol..." à la Vieille Charité. De cette rencontre naissent des œuvres réalisées par les détenus et une exposition présentée à la fin du projet au ministère de la Justice à Paris.

27 La liste des projets n'est pas close ; pour autant, malgré la présence d'un maillage dense des patrimoines sur le territoire national, le nombre d'actions menées en direction des personnes placées sous main de justice reste modeste. Une soixantaine de projets ont pu être répertoriés à partir de données recensées en 2013 et 2014 : les données ont été 
collectées à partir de l'étude de Delphine Saurier, d'une enquête interne faite auprès des établissements publics nationaux en 2011-2012 et d'informations communiquées par le Secrétariat général du ministère de la Culture et de la Communication; elles ne constituent pas cependant une photographie complète des actions culturelles patrimoniales en milieu pénitentiaire et ouvert.

CEuvres de détenus, atelier de cartes postales au Centre pénitentiaire de Nantes, en 2013

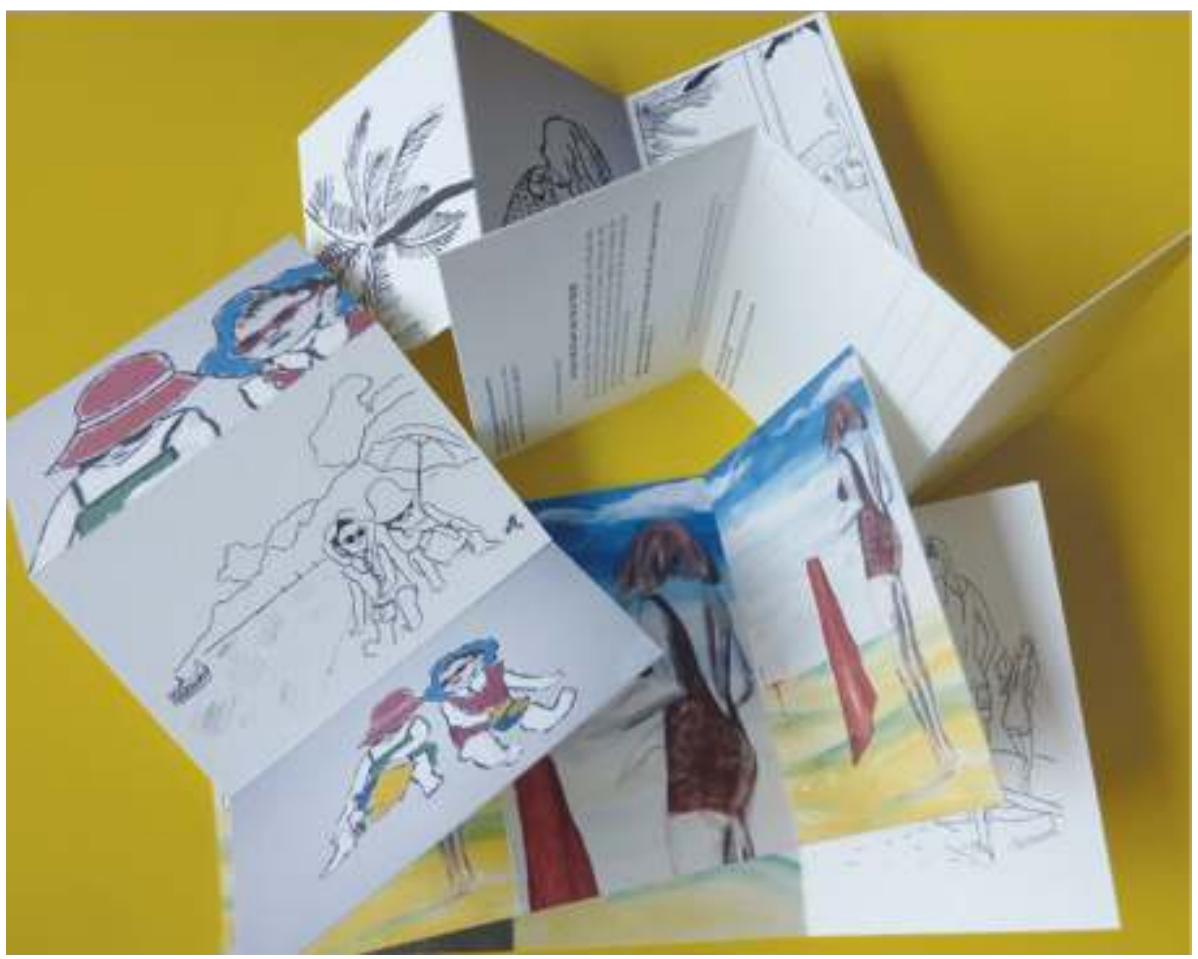

(c) Musée des Beaux-Arts de Nantes

On estime que ces projets ont touché près de 3000 personnes. Les mineurs sont autant concernés que les personnes majeures. Les hommes sont en revanche plus nombreux que les femmes, de même les personnes rattachées au milieu ouvert. Le lieu où se déroulent les projets est principalement l'établissement pénitentiaire ou l'établissement éducatif pour les jeunes sous PJJ. Les activités les plus courantes proposées sont des ateliers de pratiques artistiques, d'écriture, voire manuels (dans le cas des métiers techniques de la culture), mais aussi des cours, des conférences, des visites guidées (souvent virtuelles par le biais de vidéos), des séances de sensibilisation pour les personnels judiciaires... Parmi les difficultés recensées, on notera le caractère occasionnel des actions, le manque de temps, le caractère compliqué des modes de financements, le manque de moyens matériels et humains, la rareté des évaluations, la limitation des sorties pour une visite des lieux patrimoniaux et, élément bloquant pour des projets à vocation pluriannuelle, l'instabilité des effectifs de détenus et la rotation des acteurs professionnels impliqués dans les actions.

Quelques réflexions

Le protocole d'accord de 2009 établit le principe d'une évaluation des actions communes. À cet effet, la circulaire interministérielle d'application du 3 août 2012, relative à la mise en œuvre du protocole Culture/Justice, décline dans son annexe une fiche d'évaluation 
définissant des indicateurs chiffrés pour mesurer l'accès aux pratiques culturelles en détention, l'investissement financier dans le projet culturel et la diversification de l'offre culturelle. Elle rappelle la nécessité de transmettre un bilan, en grande partie quantitatif, sur la politique partenariale, les formations interministérielles et l'implication des institutions culturelles. L'évaluation de l'impact des actions sur les publics n'est exigée que pour le secteur de la protection judiciaire des jeunes. La fiche ne donne cependant pas le mode d'emploi de ce type d'évaluation. À l'évidence, c'est la logique du chiffre qui transparaît dans les directives du protocole. En réalité, la question de l'évaluation des projets, abordée lors de la formation par un des intervenants (Delphine Saurier), comporte plusieurs problématiques. D'abord, que faut-il évaluer ? L'accès à la culture du plus grand nombre? Les effets des actions sur les détenus? L'effectivité de la réinsertion sociale? La perception du dispositif par les acteurs impliqués? Les objectifs professionnels, citoyens, individuels, institutionnels... qui ont porté l'action ? L'évaluation peut être tout cela à la fois et de ce fait ne peut être réduite à la production d'un simple bilan chiffré. Elle suppose au contraire qu'on porte un regard rétrospectif sur le processus de l'action, qu'on interroge la question du public empêché, qu'on interroge cette rencontre singulière entre le monde de la culture et de la justice.

31 D'autres questions ont été abordées dans le cadre de la formation. Notamment, celles ayant trait à la perception du projet culturel par les acteurs impliqués. Les échanges relevés avec les stagiaires confirment les logiques, pas nécessairement antagonistes, à l'œuvre dans le projet collectif. D'une part, pour l'institution culturelle, "une conception de la culture comme porteuse d'un potentiel de socialisation"6 et la volonté d'apporter la culture en prison pour combattre l'inégalité sociale constatée d'accès au patrimoine; d'autre part, pour l'institution pénitentiaire, une perception de la culture considérée comme un outil pertinent pour la réinsertion future des détenus mais aussi comme contribuant à l'adoucissement de la peine. Les personnels pénitentiaires sont du reste placés devant une contradiction originelle, celle du rôle paradoxal de la prison. Cette dernière est à la fois envisagée comme le lieu de punition du coupable et comme le lieu de sa réadaptation sociale. Cette contradiction participe-t-elle aux freins observés dans les centres de détention pour la mise en place des projets culturels? Ne témoigne-t-elle pas de l'absence d'une réelle politique de réinsertion, malgré la volonté affirmée, dans les accords interministériels, d'ouvrir le milieu carcéral sur la société? Comme l'indique la sociologue Delphine Saurier, une "nécessaire réflexion supra sur la prise en charge des personnes condamnées et sur la place de la culture au sein de la société doit être développée, si l'on veut trouver des solutions aux problèmes divers identifiés et s'engager dans les multiples axes de progression"7.

\section{Conclusion}

La formation "Faire vivre la culture en milieu pénitentiaire" est, peut-être, l'amorce d'un processus visant à développer une culture et une méthodologie de projets communes entre des acteurs animés par des convictions souvent convergentes, mais aussi des objectifs professionnels et des impératifs institutionnels divergents. Le bilan du stage (établi sur la base d'un questionnaire d'évaluation du stage par les participants et les échanges de fin de stage), s'il laisse apparaitre une satisfaction générale, tant en termes organisationnels qu'en termes d'attentes, laisse émerger quelques réserves sur la densité des contenus pédagogiques et la concentration des séances sur une durée estimée trop courte ( 5 jours). La présence d'intervenants issu du milieu carcéral, pouvant exposer le point de vue des personnels ou des acteurs pénitentiaires, a été notamment jugée 
insuffisante même si l'intervention d'une juge d'application des peines a pu être très appréciée et permettre des échanges très constructifs. Les organisateurs regrettent par ailleurs de n'avoir pas suffisamment affiné les questions relatives aux levées de fonds et aux montages des budgets, essentiels pour la conduite des actions. Il restera également à poursuivre la réflexion sur le sens de la peine et de la culture pour mieux légitimer l'action culturelle en faveur de la population pénale et renverser les préjugés et les réticences. Il conviendra également d'illustrer davantage les modes d'évaluation et la méthodologie d'enquête qualitative. Des réajustements et des améliorations sont donc attendus mais le succès de cette formation devrait encore encourager les professionnels de la culture et de la justice à démultiplier ce type d'expérimentation. Si elles n'ont pas totalement engagé la "nécessaire réflexion" souhaitée par Delphine Saurier, la Direction générale des patrimoines et l'Enap peuvent, en tout cas, se satisfaire d'avoir initié une dynamique nationale en ce sens.

\section{Effets sur les projets de stagiaires : l'exemple du projet d'exposition Dedans Dehors.}

\section{Hervé Di Rosa présente 11 artistes de la Maison Centrale de Saint-Maur, à Issoudun}

Patrice Moreau, conservateur au musée de L'Hospice Saint-Roch d'Issoudun (Indre), a participé à l'organisation du 14 février au 17 juin 2015 d'une exposition innovante autour de l'œuvre d'Hervé Di Rosa, un des artistes fondateurs du mouvement de la «figuration libre » dans les années 1980. Le projet, dont est issue l'exposition, et à partir duquel les stagiaires vont travailler, est à l'initiative du SPIP et du coordonnateur culturel de la Fédération des organisations laïques d'Indre (FOL 36), chargés de favoriser au travers d'un atelier de peinture le lien dedans-dehors dans la Maison centrale de Saint-Maur. La rencontre entre Hervé Di Rosa et les personnes placées sous main de justice autour du concept d'art modeste aboutit à la réalisation par ces personnes d'œuvres totalement inspirées par l'univers de l'artiste, peuplé de figures d'une mythologie toute personnelle (BD, télévision, science-fiction). Les créations des détenus sont exposées pour la première fois hors les murs sur les cimaises du musée de l'Hospice Saint-Roch, en écho à celles conçues pour l'occasion par Hervé Di Rosa. La démarche qui caractérise ce projet est de considérer la Maison centrale comme un lieu à l'intérieur duquel sont vécus les mêmes enjeux culturels et artistiques qu'à l'extérieur. La réussite d'un tel projet tient à cette conjonction effectuée entre les œuvres d'Hervé Di Rosa et celles des détenus, mais aussi plus largement à l'interpénétration de deux espaces de vie et de socialisation distincts - la ville et le lieu de détention - et l'ouverture au regard sur l'autre. 


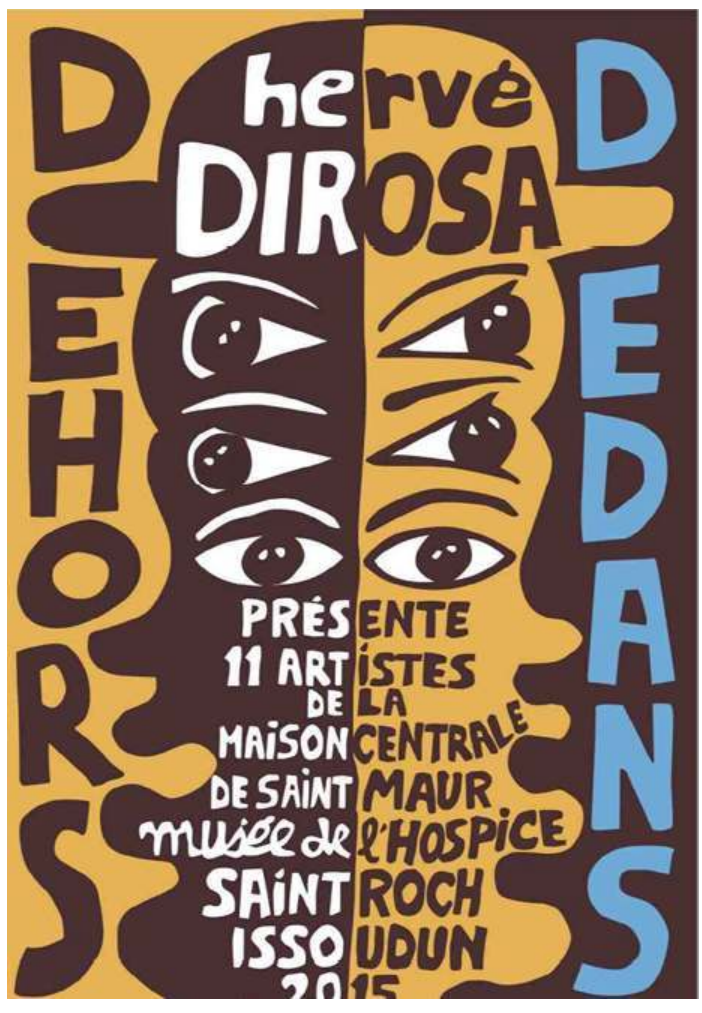

\section{BIBLIOGRAPHIE}

Centre de recherches sociologiques sur le droit et les institutions pénales (CESDIP) :

www.cesdip.fr, rubrique : Publications, Questions pénales, L'action culturelle en faveur de la population pénale par Delphine Saurier et Kathia Barbier, 3 juillet 2012.

Ministère de la culture et de la communication :

www.culturecommunication.gouv.fr

Rubrique : Politiques ministérielles, Connaissance du patrimoine et de l'architecture, Connaissance des publics, Publics et patrimoines, PatrimoÉtudes, L'action culturelle en faveur de la population pénale par Delphine Saurier et Katia Barbier, Rapport d'étude, septembre 2011

Siganos, F. L'action culturelle en prison. Paris : L'Harmattan, Série études culturelles, 2008, 270 p.

Collectif Entre les murs/hors les murs, Cultures \& Musées, n² 26, Actes Sud, janvier 2016, 256 p.

\section{NOTES}

1. L'Enap, établissement public placé sous la tutelle du ministère de la Justice, assure la formation initiale et continue de l'ensemble des personnels pénitentiaires répartis en cinq filières : personnels de direction, d'insertion et de probation, de surveillance, administratifs et techniques. 
2. Loi d'orientation du 29 juillet 1998 relative à la lutte contre les exclusions, dont l'article 140 prévoit que "l'égal accès de tous, tout au long de la vie, à la culture, à la pratique sportive, aux vacances et aux loisirs, constitue un objectif national" ; article D.158 du code de procédure pénale relatif au régime de détention des mineurs qui prévoit que le mineur détenu doit avoir "accès à des activités socioculturelles et sportives ou de détente adaptées à son âge", articles D.443 à D.449-1 qui prévoient l'accès des personnes détenues aux activités culturelles et socioculturelles.

3. Convention de l'UNESCO sur la diversité culturelle ratifiée par la communauté européenne le 18 décembre 2006 ; règles pénitentiaires européennes 27.1 à 27.7 relatives à l'exercice physique et aux activités récréatives et 28.5 à 28.6 relatives à l'éducation, adoptées le 11 janvier 2006 par la France et l'ensemble des États membres du Conseil de l'Europe, et qui prévoient l'accès aux activités culturelles et à la bibliothèque.

4. ) Étude menée par Delphine Saurier, enseignante chercheuse à Sciencescom, École de la communication et des médias, Audencia Group de Nantes.

5. Le milieu ouvert concerne les personnes qui ne sont pas sous écrou, bénéficiant de mesures alternatives à l'incarcération (sursis avec mise à l'épreuve, travail d'intérêt général, libération conditionnelle, contrôle judiciaire ou ajournement avec mise à l'épreuve) ou faisant l'objet d'un aménagement de peine en placement à l'extérieur, en semi-liberté ou en placement sous surveillance électronique

6. ) Enquête qualitative Évaluer un dispositif artistique et culturel en milieu pénitentiaire autour du partenariat entre le musée du Louvre, le SPIP de Paris et la Maison d'arrêt de la Santé. Musée du Louvre, Direction de la politique des publics et de l'éducation artistique, Service études et recherche, janvier 2010.

7. Cf. supra, Bibliographie, L'action culturelle en faveur de la population pénale.

\section{RÉSUMÉS}

Cette contribution propose un retour sur une formation innovante destinée aux professionnels de la culture et de la justice et organisée afin que les acteurs de ces deux univers - l'un patrimonial, l'autre pénitentiaire - puissent se rencontrer et échanger autour de projets culturels communs.

\section{INDEX}

Mots-clés : formation

\section{AUTEUR}

\section{MORRAD BENXAYER}

adjoint au chef du Département de la politique des publics à la Direction des patrimoines du ministère de la Culture et de la Communication

morrad.benxayer@culture.gouv.fr 